\title{
Biology of mammalian mobile DNA
}

\section{Haig H Kazazian*, Daria Babushok, Christine Courtney, Hiroki Kano, Eric Ostertag, Maria del Carmen Seleme and Nuo Yang}

\author{
Address: Department of Genetics, University of Pennsylvania School Of Medicine, Philadelphia, Pennsylvania, USA \\ * Corresponding author
}

from 2006 International Meeting of The Institute of Human Virology

Baltimore, USA. 17-2I November, 2006

Published: 21 December 2006

Retrovirology 2006, 3(Suppl I):S56 doi:I0.II86/I742-4690-3-SI-S56

(C) 2006 Kazazian et al; licensee BioMed Central Ltd.

LINE-1 or L1 retrotransposons have accounted for $1 / 3$ of the genomes of humans and other mammals through direct insertion and mobilization of other DNAs, such as Alus and processed pseudogenes. In this talk, I discuss two recent "surprises" in our study of L1 biiology. First, about $100 \mathrm{~L} 1 \mathrm{~s}$ are able to retrotranspose by a "copy and paste" mechanism in the average human genome. However, a small number of "hot" L1s account for most of this activity. Recently, we found that sequence polymorphisms have "cooled off" about a third of the "hot" L1 alleles, and that these sequence polymorphisms along with presence/ absence polymorphisms lead to considerable genetic variation in retrotransposition capability among individual humans. We estimate that the variation in retrotransposition capability among humans is up to 400 -fold. Second, evidence from genome sequence and human insertions previously led us to believe that the bulk of retrotransposition occurred in male and female germ cells, although some retrotransposition was thought to occur in early embryonic development. Now we have used mice carrying an L1 transgene driven by its endogenous promoter, and found that most, if not all, retrotransposition events occur in early embryonic development in these animals. In fact, the bulk of new insertions in these transgenic mice are not passed from generation to generation. In addition, the rate of retrotransposition in these mice does not correlate with the methylation status of the L1 promoter. Finding substantial individual variation in retrotransposition capability and extensive retrotransposition during mammalian development has had a profound effect on our thinking about the role of these mobile DNAs in genome evolution and human development. 